\title{
Generoll Entomology
}

\section{Host plants and distribution records of lance flies (Diptera: Lonchaeidae) in São Paulo State, Brazil}

\author{
Ester Marques de Sousa ${ }^{\circledR} \odot$, Léo Rodrigo Ferreira Louzeiro ${ }^{1}$, Pedro Carlos Strikis ${ }^{2}{ }^{\circledR}$, \\ Miguel Francisco de Souza-Filho ${ }^{1 \oplus}$ \& Adalton Raga ${ }^{1 \bowtie}$
}

1. Laboratório de Entomologia Econômica, Instituto Biológico, Campinas, SP, Brazil. 2. Independent Researcher, Americana, SP, Brazil.

\section{EntomoBrasilis 14: e942 (2021)}

\section{Edited by:}

Ricardo Adaime da Silva

Article History:

Received: 05.iii.2020

Accepted: 20.iv.2021

Published: 21.v.2021

$\bowtie$ Corresponding author:

Adalton Raga

७ adalton.raga@sp.gov.br

Funding agencies:

$\checkmark$ Coordenação de Aperfeiçoamento

de Pessoal de Nível Superior

\begin{abstract}
The knowledge of host plants, distribution and economic importance of Lonchaeidae is scarce in Latin America. We have recovered specimens of Lonchaeidae from most fruit samples containing specimens of Tephritidae. The compilation of information is essential to determine the diversity of species and the relationship with their hosts. In addition to the list of records based on early publications, we add unpublished data of Lonchaeids recovered from plant samples collected in the Instituto Biológico, São Paulo, Brazil. In total, 18 species of Lonchaeidae, belonging to the genera Dasiops, Lonchaea and Neosilba were registered in São Paulo, and associated with 111 host plant species and 27 botanical families. New records are listed and geographical distribution is available by specific maps.
\end{abstract}

Keywords: Insecta; Tephritoidea; Neosilba; Dasiops; fruit hosts.
Tre The Lonchaeidae family (lance flies) comprises an important group of fruit flies. Several species are associated with the decomposition of organic matter of plant origin. However, some species are considered of economic importance because they attack fruits and flower buds (McAlpINE \& SteYSKAL 1982). Species of the genera Neosilba and Dasiops are reported as primary invaders of certain fruit trees, obtaining pest status for some plant species of Euphorbiaceae, Myrtaceae, Rutaceae, Sapotaceae and Passifloraceae (UCHÔA \& Nić́cIO 2010; RAGA et al. 2011; GISLOTI et al. 2017; AdAime et al. 2017). Some lance fly species are polyphagous, and few species are known to be monophagous or oligophagous (STRIKIS 2011).

In around the State of São Paulo, Brazil, the distribution, infestation rate and infestation period of fruit flies (Tephritoidea) vary due to different agroecossystems, climatic diversity, peculiar phytophysiognomy and, the origin of the fruit sample (rural or urban region) (RAGA et al. 2011).

Regional research is crucial to provide basic information for the management of insect pest populations (UCHÔA et al. 2002). Therefore, it is important to examine historical and current research data on lance flies (Lonchaeidae), their distribution and host plants to help understanding the population dynamics of this insect group insects and developing management strategies.

The first reports of Lonchaeidae associated with fruits in São Paulo occurred when Hempel (1901) associated Lonchaea glaberrima Wiedemann [Neosilba glaberrima (Wiedemann)] with passion fruit Passiflora sp. However, the main reports of association of lance flies with host fruits, occurred in the 1980s, when dozens of species of host fruits were listed for several Brazilian states, including São Paulo (MaLAVASI et al.
1980; MalaVasi \& Morgante 1980).

Here, after 30 years of random fruit collections in different regions of São Paulo, we compile the state records of lonchaeids, based on all available publications of plant hosts, monitoring, distribution, species descriptions, and unpublished records. The present study reinforces the biological diversity and the economic importance of Lonchaeidae species within superfamily Tephritoidea.

\section{MATERIAL AND METHODS}

An extensive search of available printed or digital publications on field-collected lance flies was carried out from 1980 to 2020. This search period was considered the resumption of scientific works on Lonchaeidae in São Paulo, after the taxon redefinition and description of many species.

For each publication, we provide the complete references on lance flies and their geographical distribution. Each study was categorized into two insect collection categories: fruit or trapping. This approach was used to relate the Lonchaeidae species with plant hosts. Data from traps helping to create specific maps. With the analysis of the data of all publications, a table of relation of lance fly/hosts and distribution maps were available.

We removed duplicate records from abstracts, conference proceedings and thesis. In addition to the list of records based on early publications, we add unpublished data of Lonchaeids recovered from fruits and flower buds collected in the Instituto Biológico, São Paulo, Brazil.

\section{RESULTS}

Eighteen species of Lonchaeidae are known to São Paulo, 
belonging to the genera Dasiops, Lonchaea and Neosilba. Currently, 111 host species of lonchaeids are identified, belonging to 27 botanical families (Table 1), from which 47 are introduced species (Table 1). Species of Neosilba are the most commonly lance flies found in São Paulo, where 15 species are reported: N. glaberrima, Neosilba pendula (Bezzi), Neosilba zadolicha McAlpine \& Steyskal, Neosilba certa (Walker), Neosilba dimidiata (Curran), Neosilba inesperata Strikis \& Prado, Neosilba perezi (Romero \& Ruppel), Neosilba laura Strikis, Neosilba bifida Strikis \& Prado, Neosilba cornophallus Strikis, Neosilba pradoi Strikis \& Lorena, Neosilba parva Hennig, Neosilba bella Strikis \& Prado, Neosilba delvechioi Strikis and Neosilba paramerolatus Strikis. Only two Dasiops species were registered: Dasiops inedulis Steyskal and Dasiops frieseni Norrbom \& McAlpine. Both species of Dasiops are associated with Passiflora edulis Sims. Lonchaea was exhibited only at the genus level according to the record of original paper.

Myrtaceae has the largest number of host plants of Lonchaeidae, followed by Rosaceae, Rutaceae, Fabaceae and Rubiaceae (Table 1). The botanical species with the greatest diversity of lance flies is Eriobotrya japonica (Thunb.) (Rosaceae), with 10 associated species (nine species of Neosilba and one Lonchaea sp.), followed by Coffea spp. with 9 species. Neosilba zadolicha is highly polyphagous species among Lonchaeids in São Paulo, where is associated with 75 hosts and 22 families.

The distribution of lonchaeids in São Paulo is represented in Figure 1. There are reports of lonchaeids in 99 municipalities of São Paulo. Neosilba pendula, N. zadolicha, N. certa, N. inesperata and $N$. glaberrima are the most widely distributed

Table 1. Lonchaeidae (Diptera: Tephritoidea) host plants ( $\mathrm{N}=$ Native; I = Introduced) in São Paulo, Brazil.

\begin{tabular}{cccc}
\hline $\begin{array}{c}\text { Botanical Family (number of } \\
\text { Lonchaeidae species) } \\
\text { Botanical species }\end{array}$ & $\begin{array}{c}\text { Native } \\
\text { Introduced }\end{array}$ & Lonchaeidae species & Reference \\
\hline
\end{tabular}

\section{Anacardiaceae (4)}

$\begin{array}{llll}\text { Lithraea molleoides (Vell.) Engl. } & \text { N } & \text { N. glaberrima; N. pendula } & \text { RAGA et al. } 2015 \\ \text { Mangifera indica L. } & \text { I } & \text { N. zadolicha } & \text { RAGA et al. } 2015 \\ \text { Spondias dulcis Parkinson } & \text { I } & \text { N. pendula } & \text { RAGA et al. } 2015 \\ \text { Spondias mombin L. } & \text { N } & \text { N. certa; N. pendula; N. zadolicha } & \text { GISLOTI et al. } 2017 \\ \text { Spondias purpurea L. } & \text { I } & \text { N. pendula; N. zadolicha } & \text { RAGA et al. 2015; GISLOTI et al. } \\ \text { Spondias tuberosa Arruda } & \text { N } & \text { N. zadolicha } & \text { 2017 } \\ \text { Spondias venulosa (Engl.) Engl. } & \text { N } & \text { N. zadolicha } & \text { GISLOTI et al. } 2017\end{array}$

\section{Annonaceae (4)}

\begin{tabular}{|c|c|c|c|}
\hline Annona coriacea Mart. & $\mathrm{N}$ & N. zadolicha; N. certa & RAGA et al. 2015 \\
\hline Annona emarginata (Schltdl.) H. Rainer & $\mathrm{N}$ & N. certa & RAGA et al. 2015 \\
\hline Annona mucosa Jacq. & $\mathrm{N}$ & N. zadolicha; N. certa & RAGA et al. 2015 \\
\hline Annona reticulata $\mathrm{L}$. & I & N. zadolicha & RAGA et al. 2015 \\
\hline Annona neosericia $\mathrm{H}$. Rainer & $\mathrm{N}$ & N. dimidiata; N. zadolicha; N. glaberrima & RAGA et al. 2015 \\
\hline Annona squamosa L. x A. cherimola Mill. & I & N. zadolicha; N. pendula & RAGA et al. 2015 \\
\hline Annona sylvatica A. St.-Hil & $\mathrm{N}$ & N. zadolicha & RAGA et al. 2015 \\
\hline Annona sylvatica A. St.-Hil & $\mathrm{N}$ & N. zadolicha; N. glaberrima & GisLotı et al. 2017 \\
\hline
\end{tabular}

Apocynaceae (1)

Hancornia speciosa Gomes

N N. zadolicha

GisLotı et al. 2017

\section{Areaceae (2)}

Bactris gasipaes Kunth

N N. zadolicha; N. glaberrima

GısLotı et al. 2017

\section{Cactaceae (2)}

Pereskia aculeata Mill.

I N. glaberrima

Hylocereus setaceus (Salm-Dyck ex DC.) Ralf Bauer

N N. zadolicha; N. glaberrima

\section{New record}

GIsLotı et al. 2017

\section{Caricaceae (1)}

Carica papaya $\mathrm{L}$.

I Lonchaeasp.

RAGA et al. 2015

\section{Caryocaraceae (2)}

Caryocar brasiliensis A. St.-Hil

N N. zadolicha; N. pendula

GIsLotı et al. 2017

\section{Combretaceae (4)}

Terminalia catappa $\mathrm{L}$.

N. zadolicha; N. inesperata; N. certa; N. RAGA et al. 2015 glaberrima

\section{Cucurbitaceae (1)}

Cucurbita maxima Duchesne

N N. zadolicha

RAGA et al. 2015

Cucurbita moschata Duchesne

I N. zadolicha


Table 1. Continue..

\begin{tabular}{lllll}
\hline \multicolumn{1}{c}{$\begin{array}{c}\text { Botanical Family (number of } \\
\text { Lonchaeidae species) } \\
\text { Botanical species }\end{array}$} & $\begin{array}{c}\text { Native } \\
\text { Introduced }\end{array}$ & Lonchaeidae species & Reference \\
\hline $\begin{array}{l}\text { C. moschata Duchesne } \\
\text { Duchesne }\end{array}$ & C. maxima & I & N. zadolicha & RAGA et al. 2015
\end{tabular}

\section{Ebenaceae (1)}

Diospyros kaki L.f.

I N. zadolicha

RAGA et al. 2015

\section{Euphorbiaceae (1)}

Manihot esculenta Crantz

N N. perezi

LOURENÇÃo 1996; GISLOTI \& Prado 2011; Raga et al. 2015

\section{Fabaceae (9)}

\begin{tabular}{|c|c|c|}
\hline Dalbergia brasiliensis Vogel & $\mathrm{N}$ & N. laura \\
\hline Inga spp. & $\mathrm{N}$ & $\begin{array}{l}\text { N. zadolicha; N. certa; N. glaberrima; N. pendula; } \\
\text { N. laura; N. bifida; N. inesperata; N. cornuphallus }\end{array}$ \\
\hline
\end{tabular}

$\begin{array}{llll}\text { Inga vera Willd. } & \text { N } & \text { N. certa; N. pendula; N. pradoi; N. zadolicha } & \text { GISLOTI et al. } 2017 \\ \text { Leucaena leucocephala (Lam.) de Wit } & \text { I } & \text { N. certa; N. pendula } & \text { RAGA et al. } 2015 \\ \text { Swartzia langsdorffii Raddi } & \text { N } & \text { N. zadolicha; N. glaberrima; N. certa } & \text { RAGA et al. } 2015\end{array}$

\section{Ginkgoaceae (1)}

Ginkgo biloba L.

I $\quad$. zadolicha

STRIKIS 2011

\section{Lauraceae (5)}

Persea americana Mill.

\section{Malpighiaceae (8)}

Bunchosia armeniaca (Cav.) DC.

Byrsonima crassifolia (L.) Kunth

Malpighia emarginata DC.

\section{Malvaceae (1)}

Gossypium hirsutum L.

\section{Moraceae (6)}

Ficus carica $\mathrm{L}$.

Ficus sp.

Morus nigra L.

\section{Musaceae (1)}

Musa x paradisiaca L. (cv. Nanica)

\section{Myrtaceae (11)}

\begin{tabular}{|c|c|c|}
\hline Acca sellowiana (O. Berg) Burret & $\mathrm{N}$ & N. zadolicha \\
\hline Campomanesia auera O. Berg & $\mathrm{N}$ & N. pradoi \\
\hline $\begin{array}{l}\text { Campomanesia guazumifolia (Cambess.) O. } \\
\text { Berg }\end{array}$ & $\mathrm{N}$ & N. pradoi; N. zadolicha \\
\hline Campomanesia phaea (O. Berg.) Landrum & $\mathrm{N}$ & N. pradoi; N. zadolicha \\
\hline Eugenia brasiliensis Lam. & $\mathrm{N}$ & Neosilba sp.; N. pradoi; N. zadolicha \\
\hline Eugenia dysenterica DC. & $\mathrm{N}$ & N. inesperata; N. pendula; N. zadolicha \\
\hline Eugenia involucrata DC. & $\mathrm{N}$ & $\begin{array}{l}\text { N. certa; N. pradoi; N. laura; N. pendula; N. } \\
\text { zadolicha }\end{array}$ \\
\hline Eugenia selloi B. D. Jacks. & $\mathrm{N}$ & N. pendula; N. zadolicha \\
\hline Eugenia leitonii D. Legrand & $\mathrm{N}$ & N. glaberrima; N. zadolicha \\
\hline Eugenia pitanga (O. Berg) Nied. & $\mathrm{N}$ & N. zadolicha \\
\hline Eugenia pyriformis Cambess. & $\mathrm{N}$ & $\begin{array}{l}\text { N. zadolicha; N. certa; N. pendula; N. pradoi; N. } \\
\text { inesperata; N. laura }\end{array}$ \\
\hline Eugenia lambertiana DC. & $\mathrm{N}$ & N. pendula; N. inesperata; N. zadolicha; N. bella \\
\hline Eugenia stipitata McVaugh & $\mathrm{N}$ & N. bella; N. pendula \\
\hline
\end{tabular}

N. zadolicha; N. certa; N. glaberrima; N. pendula; RAGA et al. 2015 N. parva

\section{N. pendula}

RAGA et al. 2015

N

N. bella; N. glaberrima; N. inesperata; N. pendula; GısLotı et al. 2017 N. pradoi; N. zadolicha

N. pendula; N. inesperata; N. zadolicha; N. certa; STRIIIS \& Lerena 2009; RAGA et N. cornuphallus; N. glaberrima; N. perezi al. 2015; GIsLOTI et al. 2017

Raga et al. 2015

N. certa; N. zadolicha; N. glaberrima; N. bifida; N. RAGA et al. 2015 cornuphallus

N. certa; N. pendula; N. zadolicha

RAGA et al. 2015

N. zadolicha

RAGA et al. 2015

RAGA et al. 2015

GISLOTI et al. 2017

GisLotı et al. 2017

GIsLotı et al. 2017

GIsLOTI et al. 2017

Raga et al. 2015; Gisloti et al. 2017

GisLotı et al. 2017

Raga et al. 2015; Gisloti el al. 2017

GisLotı et al. 2017

Raga et al. 2015

GisLotı et al. 2017

Raga et al. 2015; Gisloti et al. 2017

RAGA et al. 2015

GisLotı et al. 2017

to be continued.. 
Table 1. Continue...

\begin{tabular}{|c|c|c|c|}
\hline $\begin{array}{c}\text { Botanical Family (number of } \\
\text { Lonchaeidae species) } \\
\text { Botanical species }\end{array}$ & $\begin{array}{l}\text { Native } \\
\text { Introduced }\end{array}$ & Lonchaeidae species & Reference \\
\hline Eugenia uniflora L. & $\mathrm{N}$ & N. bella; N. pendula; N. inesperata; N. zadolicha & $\begin{array}{l}\text { RAGA et al. 2015; GisLotI et al. } \\
2017\end{array}$ \\
\hline Myrciaria dubia (Kunth) McVaugh & $\mathrm{N}$ & N. zadolicha & GisLotı et al. 2017 \\
\hline Plinia cauliflora (Mart.) Kausel & $\mathrm{N}$ & N. certa & RAGA et al. 2015 \\
\hline $\begin{array}{l}\text { Myrciaria glazioviana (Kiaersk.) G. M. } \\
\text { Barroso ex Sobral }\end{array}$ & $\mathrm{N}$ & N. inesperata; N. pendula; N. certa & RAGA et al. 2015 \\
\hline Plinia edulis (Vell.) Sobral & $\mathrm{N}$ & N. bifida & RaGA et al. 2015 \\
\hline Psidium cattleianum Afzel. ex Sabine & $\mathrm{N}$ & $\begin{array}{l}\text { N. certa; N. inesperata; N. pendula; N. pradoi; N. } \\
\text { bifida; N. dimidiata; N. zadolicha }\end{array}$ & $\begin{array}{l}\text { RAGA et al. 2015; GIsLotı et al. } \\
2017\end{array}$ \\
\hline Psidium guajava L. & $\mathrm{N}$ & $\begin{array}{l}\text { N. zadolicha; N. pendula; N. certa; N. glaberrima; } \\
\text { N. bifida; N. cornuphallus; N. inesperata; N. bella; } \\
\text { N. dimidiata }\end{array}$ & $\begin{array}{l}\text { RAGA et al. 2015; Gisloti el al. } \\
2017\end{array}$ \\
\hline Psidium guineense Sw. & $\mathrm{N}$ & N. pendula; N. zadolicha & GısLotı et al. 2017 \\
\hline Syzygium jambos (L.) Alston & I & N. pendula; N. zadolicha & RAGA et al. 2015 \\
\hline $\begin{array}{l}\text { Syzygium samarangense (Blume) Merr. \& L. } \\
\text { M. Perry }\end{array}$ & 1 & N. pendula; N. certa & RAGA et al. 2015 \\
\hline
\end{tabular}

\section{Oxalidaceae (6)}

$\begin{array}{ll}\text { Averrhoa carambola L. } & \text { N. certa; N. inesperata; N. pendula; N. glaberrima; RAGA et al. } 2015 \\ \text { N. bella }\end{array}$

\section{Passifloraceae (7)}

$\begin{array}{lll}\text { Passiflora alata Curtis } & \text { N } & \begin{array}{l}\text { N. zadolicha; N. glaberrima; N. certa; Lonchaea RAGA et al. } 2015 \\ \text { sp.; Dasiops inedulis }\end{array} \\ \text { Passiflora edulis Sims } & \text { N } \quad \begin{array}{l}\text { N. zadolicha; N. certa; N. inesperata; Lonchaea RAGA et al. } 2015 \\ \text { sp.; Dasiops inedulis; D. frieseni }\end{array}\end{array}$

\section{Rhamnaceae}

Ziziphus joazeiro Mart.

N N. pendula

RAGA et al. 2015; GISLOTI et al. 2017

\section{Rosaceae (10)}

Eriobotrya japonica (Thunb.) Lindl.

N. pendula; N. certa; N. zadolicha; N. glaberrima; StrIKIS \& PRADO 2005; StRIIIS \& N. inesperata; N. bella; N. bifida; N. pradoi; N. Prado 2009; Raga et al. 2015 cornuphallus; Lonchaea sp.

Malus domestica Borkh.

N. zadolicha; N. certa; N. pendula

RAGA et al. 2015

Prunus mume (Siebold) Siebold \& Zucc.

N. certa

RAGA et al. 2015

Prunus persica (L.) Batsch.

N. zadolicha; N. certa; N. pendula; N. inesperata; RAGA et al. 2015

Prunus salicina Lindl. N. glaberrima; N. bifida; Lonchaea sp.

Pyrus communis L.

N. certa; N. pendula; N. inesperata

RAGA et al. 2015

Rubus urticifolius Poir.

N. certa

Rubus sp.

N. inesperata; N. pendula

RAGA et al. 2015

Rubiaceae (9)

Coffea spp.

N. zadolicha; N. pendula; N. certa

GisLotı et al. 2017

RAGA et al. 2015

Rutaceae (9)

$\begin{array}{lll}\text { Citrus x aurantium L. } & \text { I } & \text { N. zadolicha; N. glaberrima } \\ \begin{array}{l}\text { Citrus x microcarpa Bunge } \\ \text { Citrus limon (L.) Osbeck }\end{array} & \text { N. delvechioi } \\ \text { Citrus mitis Blanco } & \text { N. zadolicha; N. glaberrima; N. pendula; N. certa } \\ \text { Citrus reticulata Blanco cv. Ponkan } & \text { I } & \begin{array}{l}\text { N. pendula; N. glaberrima; N. zadolicha; N. certa; } \\ \text { N. laura; N. inesperata }\end{array} \\ \text { Citrus reticulata Blanco cv. Cravo } & \text { I } & \text { N. zadolicha; N. pendula; N. inesperata } \\ \text { C. reticulata Blanco x C. sinensis (L.) Osbeck } & \text { I } & \text { N. zadolicha; N. glaberrima; N. pradoi; N. parva } \\ \text { Citrus sinensis (L.) Osbeck } & \text { I } & \text { N. zadolicha; N. certa } \\ & \text { I } & \text { N. zadolicha; N. glaberrima; N. certa; N. pendula; } \\ & & \text { N. inesperata; N. bifida }\end{array}$

N. pendula; N. zadolicha; N. inesperata; N. RAGA et al. 1997; StRIKIS 2011; bella; N. certa; N. bifida; N. laura; N. delvechioi; RAGA et al. 2015 Lonchaea sp.

Lonchaea sp.


Table 1. Continue...

\section{Botanical Family (number of \\ Lonchaeidae species)}

Botanical species

\section{Native \\ Introduced}

Lonchaeidae species

Reference

Fortunella sp.

।

N. zadolicha; N. certa; N. pendula

RAGA et al. 2015

\section{Salicaceae (2)}

Dovyalis abyssinica (A. Rich) Warb. X I

N. zadolicha; N. pendula

New records Dovyalis hebecarpa (Gardner) Warb.

\section{Sapotaceae (7)}

$\begin{array}{llll}\text { Chrysophyllum cainito L. } & \text { I } & \text { N. zadolicha; N. pendula } & \text { RAGA et al. } 2015 \\ \text { Chrysophyllum mexicanum Brandegee } & \text { I } & \text { N. zadolicha } & \text { RAGA et al. } 2015 \\ \text { Manilkara zapota (L.) P. Royen } & \text { I } & \text { N. zadolicha } & \text { RAGA et al. } 2015 \\ \text { Mimusops balata (Aubl.) C. F. Gaertn. } & \text { I } & \text { N. glaberrima; N. certa } & \text { RAGA et al. } 2015 \\ \text { Pouteria caimito (Ruiz \& Pav.) Radlk. } & \text { N } & \begin{array}{l}\text { N. zadolicha; N. glaberrima; N. pendula; N. bella; } \\ \text { N. certa }\end{array} & \text { RAGA et al. 2015; GISLOTI et al. } \\ \text { Pouteria torta (Mart.) Radlk. } & \text { N } & \begin{array}{l}\text { N. dimidiata; N. zadolicha; N. glaberrima; N. RAGA et al. } 2015 \\ \text { paramerolatus }\end{array}\end{array}$

Solanaceae (8)

Capsicum sp.

Capsicum annuum L.

Mandragora officinarum $\mathrm{L}$.

Solanum aethiopicum L.

Solanum lycopersicum L.

Solanum lycopersicum var. cerasiforme

Solanum mammosum L.

Solanum melongena $\mathrm{L}$.

Solanum variabile Mart.

Verbenaceae (4)

Citharexylum myrianthum Cham.
N. glaberrima; N. certa; N. zadolicha; N. pendula; RAGA et al. 2015 N. inesperata; N. laura

N. parva; N. zadolicha; N. certa; N. glaberrima; RAGA et al. 2015 N. pendula

Neosilba sp.

RAGA et al. 2015

N. zadolicha; N. parva; N. certa; N. pendula; N. StRIKIS \& Prado 2005; RAga et glaberrima; N. pradoi; N. inesperata al. 2015

N. zadolicha; N. certa

N. certa; N. parva

RAgA et al. 2015

\section{New records}

RAGA et al. 2015

Strikis \& Prado 2005; Raga et al. 2015

RAGA et al. 2015 in the state, where they were registered on $64,59,40,32$ and 26 municipalities, respectively.

\section{DISCUSSION}

Until the 1980s, lance flies were neglected and often discarded in surveys of frugivorous flies in Brazil, due to a lack of taxonomic knowledge and, mainly because they were not considered fruit pests (GATTELLI et al. 2008). There has been a significant increase in the number of studies related to the Lonchaeidae family since the 1990s.

RAGA et al. (2011) recovered Lonchaeidae from the majority of Tephritidae host plants collected in São Paulo, and concluded that it is not an opportunistic group, although fruits with previous infestation by tephritids showed physical-chemistry changes and facilitate further lonchaeid infestation.

An extensive survey of fruit samples in 94 municipalities of São Paulo was performed by RAGA et al. (2015), totalling 113 botanical species related to 31 plant families. Lonchaeidae species were found in 77 plant species, corresponding to $68 \%$ of the plant species collected. GisLotı et al. (2017) sampled fruits from 35 species and found that almost $90 \%$ of the sampled plants were colonized by Neosilba species. Although most of the bibliography mentioned in the present study recorded the occurrence of species of lonchaeids in plants of economic importance, some native botanical species were sampled in places bordering the conservation areas.
Neosilba zadolicha, N. pendula, N. inesperata and, N. glaberrima are frequently recovered in fruit samples (UchÔA et al. 2012; Lemos et al. 2015; RAgA et al. 2015). All mentioned species occur in São Paulo all year long (RAga et al. 2015). Neosilba perezi occurs in cassava sprouts (LoURENção et al. 1996; GISLOTI \& PRADO 2011). Polyphagous species have a wider geographic distribution than species considered to be specialists, in many cases exhibit niche overlap, and many species can infest the same host (Malavasi \& Morgante 1980; Raga et al. 2011). The polyphagia of Neosilba pendula and N. zadolicha represents high adaptation to introduced plant species, being responsible for their wide geographic distribution in the state of São Paulo (Figure 1). Twenty-four plant species reported here are both hosts of $N$. zadolicha and Ceratitis capitata (Wied.) (Tephritidae) in São Paulo. Neosilba zadolicha exhibits dominance similar to C. capitata in urban areas (RAGA et al. 2011).

Lonchaeidae's knowledge in São Paulo is not yet consolidated. It is necessary to continue efforts to learn about the diversity of Lonchaeidae species, their host plants, and specially the economic losses in horticulture crops, as well as an increase in studies on biology and behaviour. The data from the present study emphasize the relevance of Lonchaeidae species to the main crops of economic importance in São Paulo. 

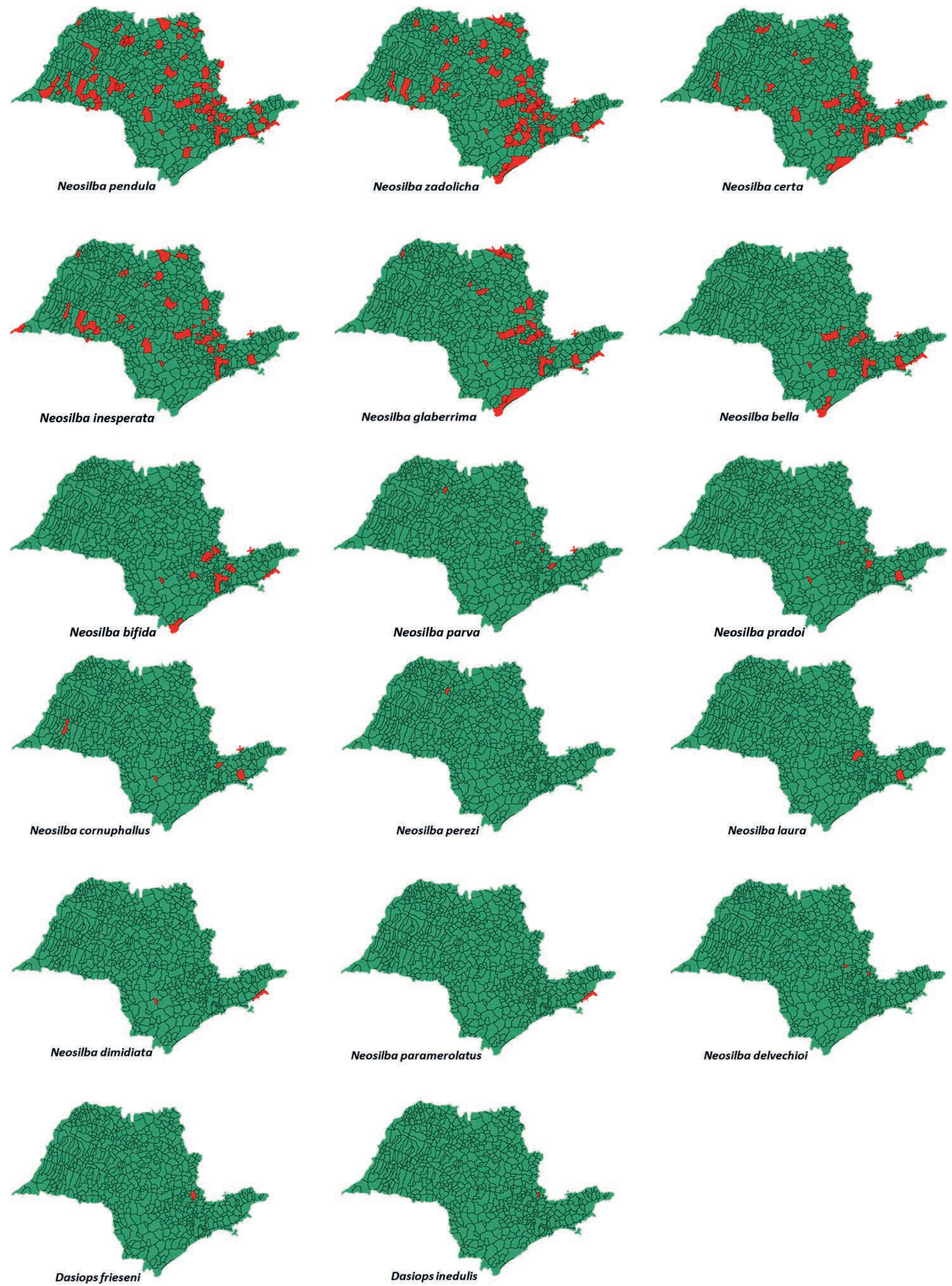

Figure 1. The known distribution of Neosilba and Dasiops species (Lonchaeidae) in São Paulo, Brazil. 


\section{REFERENCES}

Adaime, R, MDSM Sousa, CR Jesus-Barros, EDGD Deus, JF Pereira, PC Strikis \& MF Souza-Filho, 2017. Frugivorous flies (Diptera: Tephritidae, Lonchaeidae), their host plants, and associated parasitoids in the extreme north of Amapá State, Brazil. Florida Entomologist, 100: 316-324. DOI: https://doi.org/10.1653/024.100.0229

Gattelli, T, FF Silva, RNMLR Redaelli \& FKD Soglio, 2008. Moscas frugívoras associadas a mirtáceas e laranjeira "Céu" na região do Vale do Rio Caí, Rio Grande do Sul, Brasil. Ciência Rural, 38: 236-239. DOI: https://doi.org/10.1590/S0103-84782008000100038

Gisloti, LJ, MA Uchoa \& A Prado, 2017. New records of fruit trees as host for Neosilba species (Diptera, Lonchacidae) in southeast Brazil. Biota Neotropica, 17: 1-6. DOI: https://doi.org/10.1590/1676-0611-bn-2016-0213

Gisloti, LJ \& AP Prado, 2011. Cassava shoot infestation by larvae of Neosilba perezi (Romero \& Ruppell) (Diptera: Lonchaeidae) in São Paulo State, Brazil. Neotropical Entomology, 40: 312-315. DOI: https://doi.org/10.1590/ S1519-566X2011000300004

Hempel, A, 1901. Notas sobre moscas das fructas. Boletim de Agricultura, 2: 162-167.

Lemos, LN, R Adaime, SV Costa-Neto, EG Deus, CR Jesus-Barros \& PC Strikis, 2015. New findings on Lonchaeidae (Diptera: Tephritoidea) in the Brazilian Amazon. Florida Entomologist, 98: 1227-1237. DOI: https://doi.org/10.1653/024.098.0433

Lourenção, AL, JO Lorenzi \& GMB Ambrosano, 1996. Comportamento de clones de mandioca em relação a infestação por Neosilba perezi (Romero \& Ruppel) (Diptera: Lonchaeidae). Scientia Agricola, 53: 304-308. DOI: https://doi.org/10.1590/S0103-90161996000200019

Malavasi, A \& JS Morgante, 1980. Biologia de" moscas-dasfrutas" (Diptera, Tephritidae). II: Índices de infestação em diferentes hospedeiros e localidades. Revista Brasileira de Biologia, 40: 17-24.

Malavasi, A, JS Morgante \& RA Zucchi, 1980. Biologia de "moscas-das-frutas" (Diptera: Tephritidae). I. Lista de hospedeiros e ocorrência. Revista Brasileira de Biologia, 40: 9-16.

McAlpine, JF \& GC Steyskal, 1982. A revision of Neosilba McAlpine with a key to the world genera of Lonchaeidae (Diptera). Canadian Entomologist, 114: 105-137. DOI: https://doi.org/10.4039/Ent114105-2
Raga, A, MF Souza Filho, V Arthur, ME Sato, LA Machado, A Batista Filho, 1997. Observações sobre a incidência de moscas-das-frutas (Diptera: Tephritidae) em frutos de laranja (Citrus sinensis). Arquivos do Instituto Biológico, 64: 125-129.

Raga, A, MF Souza-Filho, PC Strikis \& SMNM Montes, 2015. Lance fly (Diptera: Lonchaeidae) host plants in the State of São Paulo, Southeast Brazil. Entomotropica, 30: 57-68.

Raga, A, MF Souza-Filho, RA Machado, ME Sato \& RC Siloto, 2011. Host ranges and infestation indices of fruit flies (Tephritidae) and lance flies (Lonchaeidae) in São Paulo State, Brazil. Florida Entomologist, 94: 787-794. DOI: https://doi.org/10.1653/024.094.0409

Strikis, PC, 2011. Description of 11 new species of genus Neosilba (Diptera: Lonchaeidae) from Brazil, its hosts and geographical distribution. Trends in Entomology, 7: 67-79.

Strikis, PC \& AP Prado, 2005. A new species of genus Neosilba (Diptera: Lonchaeidae). Zootaxa, 828: 1-5.

Strikis, PC \& AP Prado, 2009. Lonchaeidae associados a frutos de nêspera, Eriobotrya japonica (Thunb.). Lindley (Rosaceae), com descrição de uma nova espécie de Neosilba (Diptera: Tephritoidea). Arquivos do Instituto Biológico, 76: 49-54.

Strikis, PC \& ML Lerena, 2009. A new species of Neosilba (Diptera: Lonchaeidae) from Brazil. Iheringia Série Zoologia, 99: 273-275. DOI: https://doi.org/10.1590/S007347212009000300006

Uchôa, MA \& J Nicácio, 2010. New records of neotropical fruit flies (Tephritidae), lance flies (Lonchaeidae) (Diptera: Tephritoidea), and their host plants in the South Pantanal and adjacent areas, Brazil. Annals of the Entomological Society of America, 103: 723-733. DOI: https://doi.org/10.1603/AN09179

Uchôa, MA, I Oliveira, RMS Molina \& RA Zucchi, 2002. Species diversity of frugivorous flies (Diptera: Tephritoidea) from hosts in the cerrado of the state of Mato Grosso do Sul, Brazil. Neotropical Entomology, 31: 515-524. DOI: https://doi.org/10.1590/S1519-566X2002000400002

Uchôa, MA, CS Caires, JN Nicácio, M Duarte, 2012. Frugivory of Neosilba species (Diptera: Lonchaeidae) and Thepytus echelta (Lepidoptera: Lycaenidae) on Psittacanthus (Santalales: Loranthaceae) In Ecotonal Cerrado-South Pantanal, Brazil. Florida Entomologist, 95: 630-640. DOI: https://doi.org/10.1653/024.095.0314

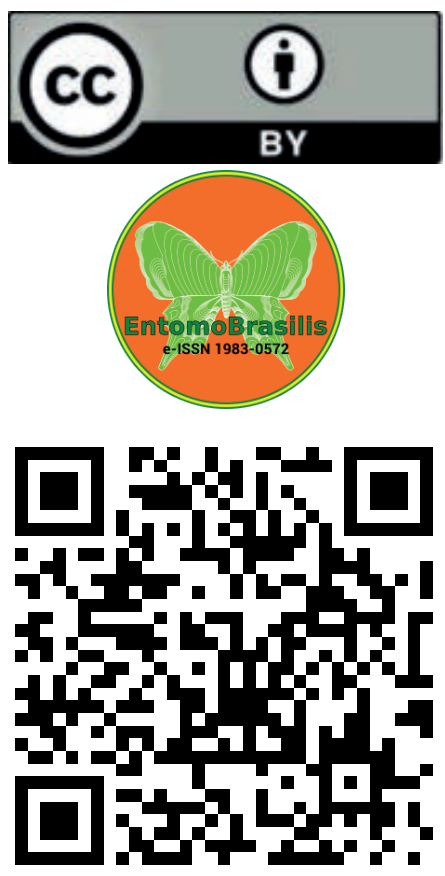

